\title{
A Study on Different Packaging Materials Affect Shelf Life of Marigold at Different Harvesting Seasons under West Bengal Condition
}

\author{
M. Preema Devi ${ }^{1}$, Laishram Hemanta ${ }^{3}$, Rocky Thokchom ${ }^{4}$, \\ S. Chakrabarty ${ }^{2}$ and R.S. Dhua ${ }^{2}$ \\ ${ }^{1}$ Department of Pomology and Post Harvest Technology, UBKV, Pundibari, \\ Cooch Behar, West Bengal, India \\ ${ }^{2}$ Department of Post Harvest Technology of Horticultural Crops, BCKV, \\ Mohanpur, Nadia, West Bengal, India \\ ${ }^{3}$ Department of Horticulture, SASRD, NU, Nagaland, India \\ ${ }^{4}$ Department of Floriculture, Medicinal and Aromatic Plants, UBKV, Pundibari, \\ Cooch Behar, West Bengal, India \\ *Corresponding author
}

\section{A B S T R A C T}

\begin{tabular}{|l|}
\hline Ke y w o r d s \\
Storage, \\
Packaging, \\
Shelf life.
\end{tabular}

\section{Introduction}

The state of West Bengal ranks $2^{\text {nd }}$ in area (6.17 Thousand $\mathrm{Ha}$ ) and $3^{\text {rd }}$ in loose marigold flower production (52.07 Thousand Metric Tonnes) in the country (NHB, 2012)). Marigold (Tagetes erecta L.) is one of the most important crops for the marginal farmers of West Bengal, who are able to earn Rs. 40,000/- per ha within four months (Anon, 2007). Uniformity in size and shape are the prime important factors for which marigold cultivars from West Bengal, mainly used as chains, are transported across the nation.
Storage of flowers is an essential part for orderly marketing and extension of shelf life. To meet the market demand on time, storage is needed to avoid glut and price fluctuation.

Appropriate methods for storing flowers even for short term are essential to provide solution during unforeseen circumstances. Focused group discussion with different stakeholders of marigold growers revealed that highest demand of loose marigold flowers in West Bengal was during October to February, 
catering the festive season, when short term storage (say a week) can offer the possibility to earn more and resist unwanted losses. Therefore, investigation on storage life of marigold was carried out during this period (early winter, mid winter and late winter) to increase the shelf life of marigold by using different packaging materials.

\section{Materials and Methods}

The experiment was conducted with three different cultivars, viz. Bidhan 1, Bidhan 2 and Bidhan 3, which were the varieties of Bidhan Chandra Krishi Viswavidyalaya (BCKV) Mohanpur, Nadia, West Bengal. Bidhan 1 was a cross between Siracole (local cultivar) and Pusa Arpita, Bidhan 2 was selected from Siracole and another improved local selection (Anonymous, 2007) was Bidhan 3.

Flowers were harvested at three different seasons i.e. early winter (Oct), mid winter (Dec) and late winter (Feb) and subsequently stored with different packaging materials (Gunny bag, nylon mesh bag, bamboo basket with cover and plastic basket with cover) during two consecutive years of 2011-12 and 2012-13, at the Laboratory of Post harvest Technology of Horticultural Crops, BCKV. Same dimensions $(13 \mathrm{~cm} \times 18 \mathrm{~cm} \times 84 \mathrm{~cm})$ were maintained in case of all the packaging materials.

Flowers were harvested at $80-100 \%$ matured stage. The temperature measured in Celsius scale and relative humidity measured by hygrometer inside the laboratory during the experiments were recorded and presented in table 1. Data pertaining to freshness of flowers, percent weight loss, dry matter content and carotenoid content (Ranganna, 2000) of the petals of marigold were observed in all the three different seasons after storage. Data were analyzed by factorial CRD by software SPSS.

\section{Results and Discussion}

\section{Shelf life}

Our experiment revealed that loose marigold flowers could be stored up to 12 days when packed in perforated plastic baskets with cover during mid winter season (Table 2). Control (i.e. without packaging) flowers had a shelf life of barely 6 days. Interaction among different factors like packaging materials, varieties and harvesting seasons varied significantly (Fig. 1) which exerted a profound influence on the shelf life of loose marigold flowers. Storing flowers under 18$20^{\circ} \mathrm{C}$ and 60 to $75 \% \mathrm{RH}$ (mid winter) was found to extend the shelf life of loose marigold flowers for extra 4-5 days (Fig. 1), compared to early winter $\left(20-22^{\circ} \mathrm{C}\right.$ and 70 to $100 \% \mathrm{RH})$ and late winter $\left(23-25^{\circ} \mathrm{C}\right.$ and 75 to $90 \% \mathrm{RH})$. The potentiality of lower temperature in extending the postharvest life of flowers has been earlier documented (Walters, 1966; Bose and Raghava, 1975), which explained that lower temperature helped to check the respiration rate and could maintain sufficient amount of carbohydrate pool needed for extending storage life. Also, packaging materials like plastic baskets with cover, facilitated air flow to create a modified atmospheric condition, a necessary prerequisite for increasing the shelf life.

\section{Physiological loss in weight (PLW)}

Temperature and relative humidity were the prime determinants of weight loss (PLW). It had a strong positive correlation with temperature and negatively correlated with relative humidity in all the cases (Table 3 ). Loose marigold flowers without packaging showed maximum loss in weight after storage in Bidhan 3 during early winter $\left(\mathrm{O}_{1} \mathrm{~V}_{3} \mathrm{~T}_{5}\right)$ while minimum loss in weight was noted in Bidhan 2 packed in plastic baskets with cover during mid winter $\left(\mathrm{O}_{2} \mathrm{~V}_{2} \mathrm{~T}_{4}\right)$, after 2 nd and 4 th day of storage (Table 4). The significant 
influence on PLW of the flowers was supported by earlier workers who mentioned that moisture content, relative water content and membrane integrity at favourable levels could delay the wilting of flowers (Ahmad et al., 2011). Increase in PLW lead to decline in fresh weight of flowers, which were expressed visually as senescing symptoms such as wilting of flowers (Nichols, 1966). Increased temperature enhanced the loss of water from the floral parts which lead to reduced flower weight. If the storage environment was more humid, the loss of water from floral parts would have been less because of high vapour pressure. Therefore, humid environment (created in plastic baskets, bags etc.) with lower temperature would minimize the loss of flower weight (Hemalatha et al., 2000).

\section{Freshness of flower}

Maximum freshness was recorded in Bidhan 2 variety packed in plastic baskets with cover during mid winter $\left(\mathrm{O}_{2} \mathrm{~V}_{2} \mathrm{~T}_{4}\right)$ at 2, 4, 6 and 8 days after storage (Table 4). Variation in weight loss and freshness of flowers during different seasons might be attributed to the lower rate of respiration of the varieties due to lower temperature during mid winter (December) as compared to other seasons. Lower rate of respiration was associated with slower rate of other enzymatic activities and subsequent delay in senescence processes (Bhattacharjee, 2001). The rate of respiration is positively correlated with storage temperature. Plastic baskets with cover acted as a barrier for loss of moisture resulting in higher relative humidity in the vicinity of the flowers and thereby less moisture loss through transpiration (Leopold and Kriedemann, 1975). Thus, rate of respiration, transpiration, ethylene production and entire metabolism of the flower tissues were retarded as a whole, facilitated by low temperature (mid winter) during storage and exhibited by better freshness of flowers.

\section{Dry matter content}

Dry matter content of loose marigold flowers showed a gradual decline throughout the storage period (Table 2). Maximum dry matter of loose marigold flowers were noted in Bidhan 2 during mid winter, packed in plastic basket with cover $\left(\mathrm{O}_{2} \mathrm{~V}_{2} \mathrm{~T}_{4}\right)$ at $2,4,6$ and 8 days after storage.

Table. 1 Variation in room temperature $\left({ }^{\circ} \mathrm{C}\right)$ and relative humidity $(\%)$ in the laboratory during early winter (October), mid winter (December) and late winter (February) for 2011 and 2012

\begin{tabular}{|c|c|c|c|c|}
\hline \multirow{2}{*}{$\begin{array}{c}\begin{array}{c}\text { Season of } \\
\text { Observation }\end{array} \\
\text { October }\end{array}$} & \multicolumn{2}{|c|}{ Temperature $\left({ }^{0} \mathrm{C}\right)$} & \multicolumn{2}{|c|}{ RH (\%) } \\
\hline & 2011 & 2012 & 2011 & 2012 \\
\hline 2DAS & 19.0 & 22 & 75 & 68 \\
\hline 4 DAS & 20.5 & 20 & 84 & 71 \\
\hline $6 \mathrm{DAS}$ & 21.0 & 21 & 100 & 76 \\
\hline $8 \mathrm{DAS}$ & 20.0 & 21 & 81 & 80 \\
\hline \multicolumn{5}{|l|}{ December } \\
\hline 2 DAS & 18 & 20 & 70 & 75 \\
\hline $4 \mathrm{DAS}$ & 18.7 & 19 & 74 & 60 \\
\hline 6 DAS & 18.6 & 19 & 72 & 60 \\
\hline 8 DAS & 18.3 & 20 & 62 & 68 \\
\hline \multicolumn{5}{|l|}{ Febuary } \\
\hline 2 DAS & 23.3 & 24 & 84 & 87 \\
\hline 4 DAS & 23.5 & 25 & 85 & 85 \\
\hline $6 \mathrm{DAS}$ & 23.7 & 25 & 84 & 78 \\
\hline 8 DAS & 23.9 & 25 & 90 & 76 \\
\hline
\end{tabular}


Table.2 Shelf life (days), Physiological loss in weight (\%), Freshness of loose marigold flowers (on 9 point hedonic scale), Dry matter content $(\%)$ and Carotenoid content $(\mu \mathrm{g} / \mathrm{g})$ of loose marigold flowers at different days after storage as affected by different packaging materials, seasons of observations and varieties

\begin{tabular}{|c|c|c|c|c|c|c|c|c|c|c|c|c|c|c|c|c|c|}
\hline \multirow[b]{2}{*}{ Treatment } & \multirow[t]{2}{*}{ Shelf life } & \multicolumn{4}{|c|}{ Physiological loss in weight (\%) } & \multicolumn{4}{|c|}{ Freshness } & \multicolumn{4}{|c|}{ Dry matter content (\%) } & \multicolumn{4}{|c|}{ Carotenoid content $(\mu \mathrm{g} / \mathrm{g})$} \\
\hline & & $\begin{array}{c}2 \\
\text { DAS } \\
\end{array}$ & $\begin{array}{c}4 \\
\text { DAS } \\
\end{array}$ & $\begin{array}{c}6 \\
\text { DAS } \\
\end{array}$ & $\begin{array}{c}8 \\
\text { DAS } \\
\end{array}$ & $\begin{array}{c}2 \\
\text { DAS }\end{array}$ & $\begin{array}{c}4 \\
\text { DAS } \\
\end{array}$ & $\begin{array}{c}6 \\
\text { DAS } \\
\end{array}$ & $\begin{array}{c}8 \\
\text { DAS } \\
\end{array}$ & $\begin{array}{c}2 \\
\text { DAS } \\
\end{array}$ & $\begin{array}{c}4 \\
\text { DAS } \\
\end{array}$ & $\begin{array}{c}6 \\
\text { DAS }\end{array}$ & $\begin{array}{c}8 \\
\text { DAS }\end{array}$ & 2 DAS & 4 DAS & 6 DAS & 8 DAS \\
\hline $\mathrm{T}_{1}$ & 9.00 & 8.68 & 14.29 & 22.72 & 27.85 & 7.00 & 6.22 & 5.64 & 5.42 & 57.05 & 49.24 & 39.79 & 35.79 & 1176.78 & 1128.22 & 1023.49 & 963.47 \\
\hline $\mathrm{T}_{2}$ & 9.97 & 7.27 & 12.74 & 20.15 & 22.32 & 7.62 & 7.06 & 6.51 & 6.00 & 61.3 & 52.24 & 44.29 & 43.61 & 1210.18 & 1129.87 & 1037.82 & 1005.21 \\
\hline $\mathrm{T}_{3}$ & 10.97 & 6.33 & 11.04 & 15.77 & 16.69 & 8.26 & 7.65 & 7.15 & 6.75 & 64.07 & 56.91 & 50.67 & 43.46 & 1288.06 & 1170.9 & 1047.59 & 1005.20 \\
\hline $\mathrm{T}_{4}$ & 12.00 & 4.38 & 8.69 & 12.4 & 14.16 & 8.75 & 8.25 & 7.75 & 7.46 & 69.16 & 61.58 & 54.81 & 49.85 & 1330.94 & 1221.08 & 1096.97 & 1040.29 \\
\hline $\mathrm{T}_{5}$ & 7.67 & 9.69 & 16.52 & 26.05 & 33.14 & 5.81 & 4.97 & 4.44 & 4.42 & 54.75 & 47.41 & 39 & 37.20 & 1147.34 & 1070.3 & 1009.03 & 956.50 \\
\hline $\operatorname{SEm}( \pm)$ & 0.055 & 0.026 & 0.044 & 0.1 & 0.150 & 0.048 & 0.048 & 0.048 & 0.059 & 0.215 & 0.215 & 0.216 & 0.284 & 0.28 & 0.28 & 0.28 & 0.55 \\
\hline $\begin{array}{c}\text { CD } \\
(P=0.05)\end{array}$ & 0.154 & 0.072 & 0.123 & 0.278 & 0.419 & 0.134 & 0.134 & 0.134 & 0.165 & 0.599 & 0.599 & 0.601 & 0.793 & 0.78 & 0.78 & 0.78 & 1.54 \\
\hline \multicolumn{18}{|c|}{ Seasons of Observation $(\mathrm{O})$} \\
\hline $\mathrm{O}_{1}$ & 10.23 & 8.45 & 10.36 & 16.16 & 21.84 & 7.42 & 6.75 & 6.40 & 5.73 & 59.06 & 55 & 48.76 & 41.51 & 1213.61 & 1128.69 & 1045.91 & 977.56 \\
\hline $\mathrm{O}_{2}$ & 11.27 & 3.22 & 8.05 & 15.79 & 23.82 & 7.92 & 7.37 & 6.90 & 6.28 & 70.36 & 60.9 & 50.28 & 42.46 & 1254.71 & 1164.8 & 1095.42 & 1010.71 \\
\hline $\mathrm{O}_{3}$ & 8.27 & 10.14 & 19.55 & 26.3 & - & 7.13 & 6.38 & 5.59 & - & 54.38 & 44.52 & 38.1 & - & 1223.66 & 1138.74 & 987.61 & - \\
\hline $\operatorname{SEm}( \pm)$ & 0.111 & 0.02 & 0.034 & 0.077 & 0.095 & 0.037 & 0.037 & 0.037 & 0.037 & 0.167 & 0.167 & 0.167 & 0.179 & 0.22 & 0.22 & 0.22 & 0.34 \\
\hline $\begin{array}{c}\mathbf{C D} \\
(\mathbf{P}=\mathbf{0 . 0 5})\end{array}$ & 0.384 & 0.056 & 0.095 & 0.214 & 0.265 & 0.103 & 0.103 & 0.103 & 0.103 & 0.465 & 0.465 & 0.465 & 0.500 & 0.6 & 0.6 & 0.6 & 0.97 \\
\hline \multicolumn{18}{|l|}{ Variety (V) } \\
\hline $\mathrm{V}_{1}$ & 9.67 & 6.26 & 11.15 & 16.62 & 21.16 & 7.05 & 6.35 & 5.83 & 5.55 & 62.31 & 54.73 & 47.78 & 42.19 & 1089.07 & 1052.28 & 965.17 & 919.17 \\
\hline $\mathrm{V}_{2}$ & 9.63 & 6.97 & 12.46 & 19.81 & 23.42 & 7.60 & 6.93 & 6.38 & 6.1 & 62.56 & 54.42 & 45 & 41.60 & 1267.68 & 1161.01 & 1044.87 & 1006.56 \\
\hline $\mathrm{V}_{3}$ & 10.47 & 8.58 & 14.35 & 21.83 & 23.92 & 7.81 & 7.21 & 6.68 & 6.38 & 58.93 & 51.28 & 44.37 & 42.16 & 1335.24 & 1218.94 & 1118.9 & 1056.68 \\
\hline $\operatorname{SEm}( \pm)$ & 0.043 & 0.02 & 0.034 & 0.077 & 0.117 & 0.037 & 0.037 & 0.037 & 0.046 & 0.167 & 0.167 & 0.167 & 0.220 & 0.217 & 0.217 & 0.217 & 0.42 \\
\hline $\begin{array}{c}\text { CD } \\
(\mathbf{P}=0.05)\end{array}$ & 0.120 & 0.056 & 0.095 & 0.214 & 0.326 & 0.103 & 0.103 & 0.103 & 0.128 & 0.465 & 0.465 & 0.465 & 0.614 & 0.604 & 0.604 & 0.604 & 1.19 \\
\hline
\end{tabular}

$\mathrm{T}_{1}$ : Gunny bags; $\mathrm{T}_{2}$ : Nylon mesh bags; $\mathrm{T}_{3}$ : Bamboo baskets with cover (perforated); $\mathrm{T}_{4}$ : Plastics baskets with cover (perforated); $\mathrm{T}_{5}$ : Control

$\mathrm{O}_{1}$ : Early winter; $\mathrm{O}_{2}$ : Mid winter; $\mathrm{O}_{3}$ : Late winter; $\mathrm{V}_{1}$ : Bidhan $1 ; \mathrm{V}_{2}$ : Bidhan $2 ; \mathrm{V}_{3}$ : Bidhan 3 
Table.3 Impact of storage environment on Physiological loss in weight for 2011, 2012 and pooled data

\begin{tabular}{|c|c|c|c|c|c|c|c|c|c|c|}
\hline & & PLW2 & PLW4 & PLW6 & Temp2 & Temp4 & Temp6 & RH2 & RH4 & RH6 \\
\hline \multirow[t]{3}{*}{ PLW2 } & Pearson Correlation & 1 & $.822^{* *}$ & $.735^{* *}$ & $.335^{* *}$ & $.285^{* *}$ & $.199^{* * *}$ & $-.721^{* *}$ & $-.736^{* *}$ & $-.523^{* *}$ \\
\hline & Sig. (2-tailed) & & .000 & .000 & .000 & .000 & .000 & .000 & .000 & .000 \\
\hline & $\mathrm{N}$ & 360 & 360 & 360 & 360 & 360 & 360 & 360 & 360 & 360 \\
\hline \multirow[t]{3}{*}{ PLW4 } & Pearson Correlation & $.822^{* *}$ & 1 & $.843^{* *}$ & $.572^{* * *}$ & $.553^{* *}$ & $.405^{* *}$ & $-.689^{* *}$ & $-.738^{* *}$ & $-.554^{* *}$ \\
\hline & Sig. (2-tailed) & .000 & & .000 & .000 & .000 & .000 & .000 & .000 & .000 \\
\hline & $\mathrm{N}$ & 360 & 360 & 360 & 360 & 360 & 360 & 360 & 360 & 360 \\
\hline \multirow[t]{3}{*}{ PLW6 } & Pearson Correlation & $.735^{* *}$ & $.843^{* *}$ & 1 & $.551^{* *}$ & $.554^{* *}$ & $.433^{* *}$ & $-.492^{* *}$ & $-.534^{* *}$ & $-.412^{* *}$ \\
\hline & Sig. (2-tailed) & .000 & .000 & & .000 & .000 & .000 & .000 & .000 & .000 \\
\hline & $\mathrm{N}$ & 360 & 360 & 360 & 360 & 360 & 360 & 360 & 360 & 360 \\
\hline
\end{tabular}

** Correlation is significant at the 0.01 level (2-tailed)

* Correlation is significant at the 0.05 level (2-tailed) 
Table.4 Physiological Loss in Weight (\%), freshness of flower and dry matter (\%) of loose marigold flowers at different days after storage as affected by different packaging materials, seasons of observations of observations and varieties

\begin{tabular}{|c|c|c|c|c|c|c|c|c|c|c|c|c|}
\hline \multirow{2}{*}{ Treatment } & \multicolumn{4}{|c|}{ PLW (\%) } & \multicolumn{4}{|c|}{ Freshness of flower } & \multicolumn{4}{|c|}{ Dry matter (\%) } \\
\hline & 2DAS & 4DAS & 6DAS & 8DAS & 2DAS & 4DAS & 6DAS & 8DAS & 2DAS & 4DAS & 6DAS & 8DAS \\
\hline $\mathrm{O}_{1} \mathrm{~V}_{1} \mathrm{~T}_{1}$ & 8.50 & 10.24 & 13.79 & 25.13 & 6.25 & 5.00 & 6.00 & 4.50 & 57.66 & 63.29 & 46.56 & 33.94 \\
\hline $\mathrm{O}_{1} \mathrm{~V}_{1} \mathrm{~T}_{2}$ & $\begin{array}{l}6.64 \\
\end{array}$ & $\begin{array}{l}8.89 \\
\end{array}$ & 13.15 & 22.79 & 7.00 & 6.50 & 7.75 & 5.25 & 61.56 & 72.23 & 50.17 & 41.72 \\
\hline $\mathrm{O}_{1} \mathrm{~V}_{1} \mathrm{~T}_{3}$ & 6.17 & 8.24 & 11.80 & 12.35 & 7.50 & 4.25 & 6.00 & 5.50 & 56.85 & \begin{tabular}{|l|l|}
53.48 \\
\end{tabular} & 51.58 & 35.81 \\
\hline $\mathrm{O}_{1} \mathrm{~V}_{1} \mathrm{~T}_{4}$ & 2.70 & 3.74 & 6.24 & 14.59 & 8.50 & 6.50 & 7.00 & 7.00 & 54.28 & 58.51 & 62.54 & $\begin{array}{l}53.74 \\
\end{array}$ \\
\hline $\mathrm{O}_{1} \mathrm{~V}_{1} \mathrm{~T}_{5}$ & $\mathbf{1 0 . 3 7}$ & 10.81 & 17.86 & 36.26 & 5.50 & 8.25 & 4.75 & 3.75 & \begin{tabular}{|c|}
69.21 \\
\end{tabular} & 52.99 & 49.13 & 39.63 \\
\hline $\mathrm{O}_{1} \mathrm{~V}_{2} \mathrm{~T}_{1}$ & 10.05 & 10.89 & 20.36 & 32.50 & 7.00 & 6.00 & 7.00 & 5.25 & 53.45 & 60.40 & 37.60 & 35.33 \\
\hline $\mathrm{O}_{1} \mathrm{~V}_{2} \mathrm{~T}_{2}$ & 8.61 & 10.82 & 15.96 & 22.71 & 7.50 & 7.50 & 8.00 & 5.75 & 57.47 & 61.00 & 44.37 & 44.69 \\
\hline $\mathrm{O}_{1} \mathrm{~V}_{2} \mathrm{~T}_{3}$ & 8.24 & 10.37 & 13.52 & 22.20 & 8.00 & 5.50 & 6.00 & 6.25 & 53.45 & 63.29 & 51.00 & 31.92 \\
\hline $\mathrm{O}_{1} \mathrm{~V}_{2} \mathrm{~T}_{4}$ & 3.81 & 6.43 & 10.62 & $\begin{array}{l}13.47 \\
\end{array}$ & 8.69 & 6.25 & 7.75 & 7.50 & 71.91 & 74.33 & 53.11 & 46.23 \\
\hline $\mathrm{O}_{1} \mathrm{~V}_{2} \mathrm{~T}_{5}$ & 10.82 & 12.84 & 21.27 & 32.50 & 6.25 & 8.25 & 5.00 & 4.25 & 76.69 & 61.56 & 44.21 & 40.25 \\
\hline $\mathrm{O}_{1} \mathrm{~V}_{3} \mathrm{~T}_{1}$ & 11.79 & 14.47 & 21.31 & 29.03 & 7.25 & 6.50 & 6.75 & 5.50 & 65.21 & 76.83 & 49.76 & 48.41 \\
\hline $\mathrm{O}_{1} \mathrm{~V}_{3} \mathrm{~T}_{2}$ & 10.81 & 13.13 & 20.02 & 24.13 & 8.00 & 8.00 & 8.00 & 6.25 & 80.79 & 85.49 & 46.71 & 42.84 \\
\hline $\mathrm{O}_{1} \mathrm{~V}_{3} \mathrm{~T}_{3}$ & 9.51 & 11.32 & 16.45 & 13.86 & 8.50 & 5.25 & 6.75 & 7.00 & 62.45 & 58.21 & 50.73 & 42.72 \\
\hline $\mathrm{O}_{1} \mathrm{~V}_{3} \mathrm{~T}_{4}$ & 5.93 & 8.11 & 13.16 & 15.75 & 8.69 & 8.00 & 7.75 & 7.50 & 64.13 & 76.69 & 52.78 & 49.97 \\
\hline $\mathrm{O}_{1} \mathrm{~V}_{3} \mathrm{~T}_{5}$ & 12.93 & 15.11 & 26.88 & 40.14 & 6.00 & 8.25 & 5.00 & 4.75 & 82.41 & 55.43 & 41.20 & 35.44 \\
\hline $\mathrm{O}_{2} \mathrm{~V}_{1} \mathrm{~T}_{1}$ & 4.49 & 8.46 & 16.65 & 25.45 & 7.00 & 4.25 & 5.00 & 5.00 & 55.48 & 54.00 & \begin{tabular}{|l|}
33.81 \\
\end{tabular} & 31.56 \\
\hline $\mathrm{O}_{2} \mathrm{~V}_{1} \mathrm{~T}_{2}$ & $\mathbf{3 . 3 6}$ & 7.68 & 13.59 & 20.04 & 7.25 & 5.50 & 7.00 & 5.50 & 58.51 & 58.74 & 48.38 & 46.06 \\
\hline $\mathrm{O}_{2} \mathrm{~V}_{1} \mathrm{~T}_{3}$ & 2.04 & 4.35 & 8.31 & 15.35 & 8.50 & 3.00 & 5.00 & 7.00 & 48.48 & 56.40 & 61.86 & 54.13 \\
\hline $\mathrm{O}_{2} \mathrm{~V}_{1} \mathrm{~T}_{4}$ & 2.64 & 5.25 & 9.64 & $\begin{array}{l}10.38 \\
\end{array}$ & 9.00 & 6.25 & 7.00 & 7.50 & 55.47 & 54.14 & 59.67 & 42.33 \\
\hline $\mathrm{O}_{2} \mathrm{~V}_{1} \mathrm{~T}_{5}$ & 5.46 & 11.77 & 22.22 & 29.31 & 6.25 & 7.00 & 3.50 & 4.50 & 61.51 & 51.65 & 48.17 & 42.98 \\
\hline $\mathrm{O}_{2} \mathrm{~V}_{2} \mathrm{~T}_{1}$ & 3.76 & 9.76 & 19.06 & 26.75 & 7.50 & 5.25 & 6.81 & 6.00 & 50.26 & 51.35 & 32.34 & 30.09 \\
\hline $\mathrm{O}_{2} \mathrm{~V}_{2} \mathrm{~T}_{2}$ & 2.32 & 6.71 & 14.50 & 21.30 & 8.00 & 7.31 & 7.25 & 6.25 & 54.67 & 55.19 & 50.69 & 40.83 \\
\hline $\mathrm{O}_{2} \mathrm{~V}_{2} \mathrm{~T}_{3}$ & 1.92 & 6.62 & 14.11 & 17.25 & 9.00 & 3.75 & 0.143 & 7.50 & 49.89 & 0.645 & 59.90 & 49.21 \\
\hline $\mathrm{O}_{2} \mathrm{~V}_{2} \mathrm{~T}_{4}$ & 0.60 & 2.63 & 13.91 & 15.45 & 9.00 & 0.398 & 5.25 & 7.50 & 1.796 & 54.85 & 65.71 & 55.93 \\
\hline $\mathrm{O}_{2} \mathrm{~V}_{2} \mathrm{~T}_{5}$ & 3.76 & 9.76 & 19.06 & 30.05 & 6.50 & 6.25 & 6.75 & 4.75 & 56.35 & $\begin{array}{l}60.72 \\
\end{array}$ & 46.70 & 41.51 \\
\hline $\mathrm{O}_{2} \mathrm{~V}_{3} \mathrm{~T}_{1}$ & 5.14 & 11.25 & 21.07 & 28.24 & 7.75 & 8.00 & 5.00 & 6.25 & 68.48 & 52.26 & 48.32 & 35.40 \\
\hline $\mathrm{O}_{2} \mathrm{~V}_{3} \mathrm{~T}_{2}$ & 3.37 & 8.58 & 15.98 & 22.97 & 8.50 & 6.25 & 7.00 & 7.00 & 52.33 & 51.83 & 49.13 & 45.55 \\
\hline $\mathrm{O}_{2} \mathrm{~V}_{3} \mathrm{~T}_{3}$ & 2.20 & 4.68 & 9.27 & 19.11 & 9.00 & 7.50 & 8.50 & 7.25 & 54.90 & $\begin{array}{l}59.19 \\
\end{array}$ & $\begin{array}{l}53.58 \\
\end{array}$ & 46.98 \\
\hline $\mathrm{O}_{2} \mathrm{~V}_{3} \mathrm{~T}_{4}$ & 1.04 & 9.12 & 11.61 & 15.34 & 9.00 & 5.25 & 6.50 & 7.75 & 46.68 & 50.12 & 55.88 & 50.88 \\
\hline $\mathrm{O}_{2} \mathrm{~V}_{3} \mathrm{~T}_{5}$ & 6.20 & 14.13 & 27.97 & 30.61 & 6.50 & 7.50 & 8.00 & 4.50 & $\mathbf{5 3 . 4 1}$ & 55.50 & 40.00 & 23.41 \\
\hline $\mathrm{O}_{3} \mathrm{~V}_{1} \mathrm{~T}_{1}$ & $\begin{array}{l}9.84 \\
\end{array}$ & 19.05 & 29.96 & - & 5.75 & 8.50 & 5.00 & - & 57.42 & 51.00 & 38.25 & - \\
\hline $\mathrm{O}_{3} \mathrm{~V}_{1} \mathrm{~T}_{2}$ & 7.61 & 16.45 & 28.24 & - & 6.75 & 6.25 & 6.50 & - & 58.72 & 58.11 & 40.25 & - \\
\hline $\mathrm{O}_{3} \mathrm{~V}_{1} \mathrm{~T}_{3}$ & 6.88 & 14.35 & 19.66 & - & 7.25 & 8.00 & 8.50 & - & 68.21 & 68.36 & 44.71 & - \\
\hline $\mathrm{O}_{3} \mathrm{~V}_{1} \mathrm{~T}_{4}$ & 5.63 & 11.68 & 11.83 & - & 8.25 & 5.50 & 7.00 & - & 54.85 & 57.25 & 50.12 & - \\
\hline $\mathrm{O}_{3} \mathrm{~V}_{1} \mathrm{~T}_{5}$ & 11.60 & 26.31 & 26.31 & - & 5.00 & 7.50 & 8.50 & - & 66.26 & 70.24 & 31.44 & - \\
\hline $\mathrm{O}_{3} \mathrm{~V}_{2} \mathrm{~T}_{1}$ & 11.63 & 21.89 & 30.25 & - & 7.00 & 8.75 & 5.75 & - & 77.80 & \begin{tabular}{|l|l|}
53.38 \\
\end{tabular} & 36.25 & - \\
\hline $\mathrm{O}_{3} \mathrm{~V}_{2} \mathrm{~T}_{2}$ & 9.85 & 21.14 & 29.61 & - & 7.50 & 7.25 & 8.25 & - & 54.10 & 58.26 & 35.47 & - \\
\hline $\mathrm{O}_{3} \mathrm{~V}_{2} \mathrm{~T}_{3}$ & 8.84 & 19.43 & 22.07 & - & 8.00 & 8.50 & 8.75 & - & 64.07 & 69.05 & 44.98 & - \\
\hline $\mathrm{O}_{3} \mathrm{~V}_{2} \mathrm{~T}_{4}$ & 7.79 & 14.79 & 16.45 & - & 8.50 & 5.50 & 5.00 & - & $\mathbf{3 4 . 3 6}$ & \begin{tabular}{|l|}
34.81 \\
\end{tabular} & 47.21 & - \\
\hline $\mathrm{O}_{3} \mathrm{~V}_{2} \mathrm{~T}_{5}$ & 12.58 & 22.86 & 36.43 & - & 5.25 & 6.00 & 6.25 & - & 40.37 & 48.12 & 25.41 & - \\
\hline $\mathrm{O}_{3} \mathrm{~V}_{3} \mathrm{~T}_{1}$ & 12.93 & 22.57 & 32.09 & - & 7.50 & 7.75 & 4.25 & - & 52.41 & 44.71 & 35.23 & - \\
\hline $\mathrm{O}_{3} \mathrm{~V}_{3} \mathrm{~T}_{2}$ & 12.92 & 21.30 & 30.33 & - & 8.06 & 6.25 & 7.00 & - & 42.21 & 42.84 & 33.46 & - \\
\hline $\mathrm{O}_{3} \mathrm{~V}_{3} \mathrm{~T}_{3}$ & 11.23 & 20.02 & 26.78 & - & 8.56 & 7.25 & 7.50 & - & 44.38 & 49.25 & 37.66 & - \\
\hline $\mathrm{O}_{3} \mathrm{~V}_{3} \mathrm{~T}_{4}$ & 9.33 & 16.45 & 18.13 & - & 8.50 & 4.00 & 6.25 & - & 47.76 & 39.20 & 46.31 & - \\
\hline $\mathrm{O}_{3} \mathrm{~V}_{3} \mathrm{~T}_{5}$ & 13.48 & 25.09 & 36.48 & - & 5.00 & 7.56 & 8.06 & - & 42.70 & 46.03 & 24.74 & - \\
\hline SEm ( \pm ) & $\mathbf{0 . 0 7 9}$ & 0.131 & 0.299 & 0.369 & 0.143 & 8.00 & 4.50 & 0.144 & 52.27 & 41.20 & 0.647 & 0.695 \\
\hline $\mathrm{CD}(\mathrm{P}=0.05)$ & 0.220 & 0.365 & 0.833 & 1.030 & 0.398 & 0.143 & 0.398 & 0.402 & 0.645 & 1.796 & 1.801 & 1.939 \\
\hline
\end{tabular}

$\mathrm{T}_{1}$ : Gunny bags; $\mathrm{T}_{2}$ : Nylon mesh Bags; $\mathrm{T}_{3}$ : Bamboo baskets with cover (perforated); $\mathrm{T}_{4}$ : Plastics baskets with cover (perforated);

$\mathrm{T}_{5}$ : Control; $\mathrm{O}_{1}$ : Early winter; $\mathrm{O}_{2}$ : Mid winter; $\mathrm{O}_{3}$ : Late winter; $\mathrm{V}_{1}$ : Bidhan $1 ; \mathrm{V}_{2}$ : Bidhan $2 ; \mathrm{V}_{3}$ : Bidhan 3. 
Fig.1 Interaction effects of different seasons of observations, varieties and packing materials on shelf life of loose marigold flowers
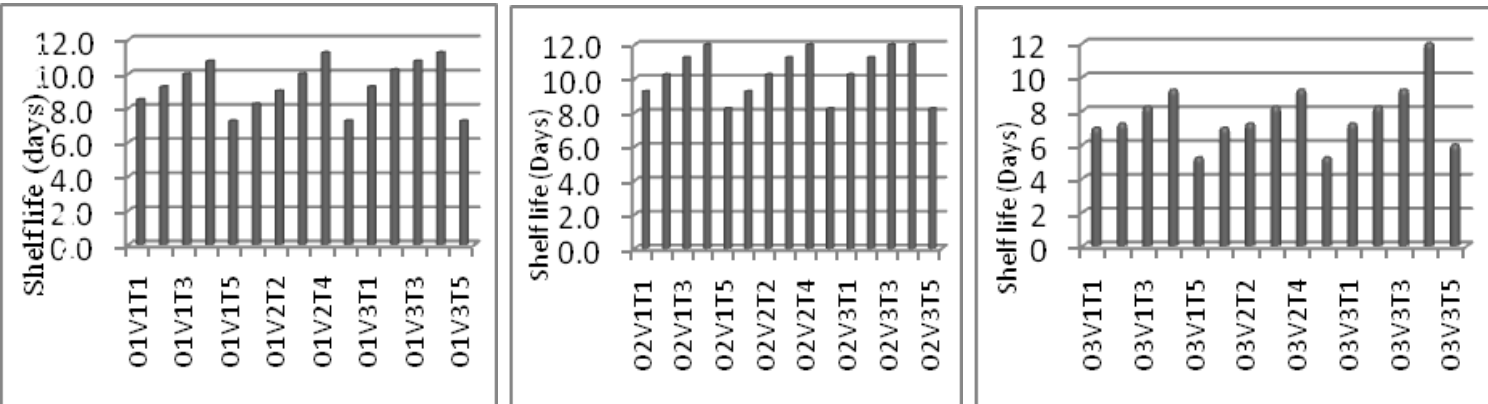

Fig.2 Interaction effects of different seasons of observations, varieties and packaging material on carotenoid content $(\mu \mathrm{g} / \mathrm{g})$ of loose marigold flowers at different days after storage

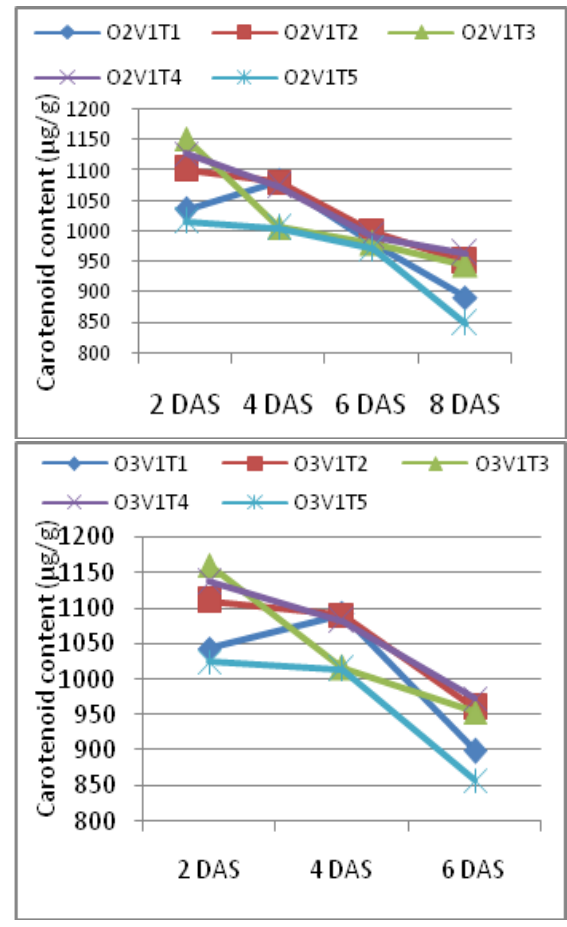

On the contrary, minimum dry matter of flowers was observed in Bidhan 3 variety without packaging stored during late winter $\left(\mathrm{O}_{3} \mathrm{~V}_{3} \mathrm{~T}_{5}\right)$ after storage (Table 4). A decline in dry matter content of the petals during final stage of flower development was reported (Nichols, 1975). Evidences supporting this fact suggested that pool of dry matter and respirable substrates were maintained at favourable levels thus promoting respiration (Croots, 1973) and became a key to extend the longevity (Rogers, 1973).
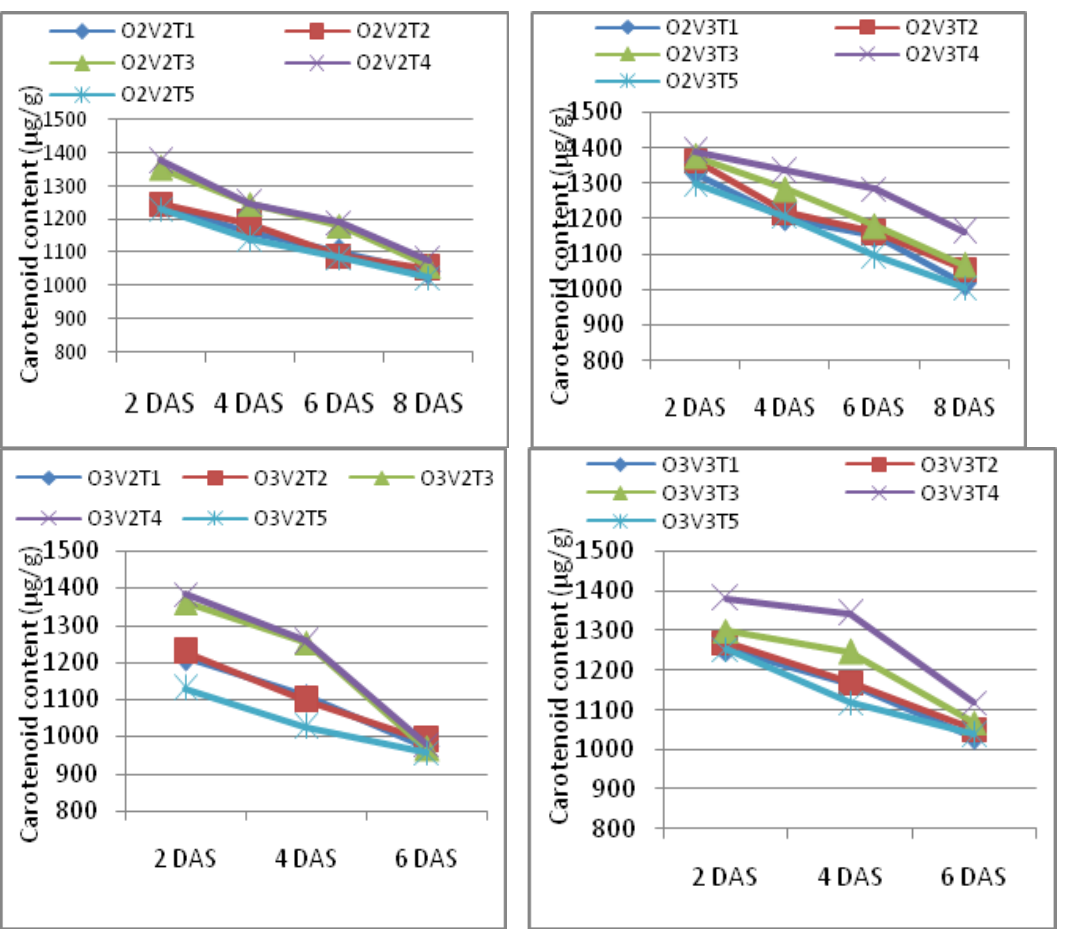

\section{Carotenoid content of the petal}

Marigold cultivars with orange flowers have higher xanthophylls as compared to yellow flowers. Therefore this was in line with our findings were maximum carotenoid content was observed in Bidhan 3 variety packed in plastic basket during mid winter for all observations after storage as illustrated in table 2 and figure 2. A gradual decreased in carotenoid content was noted for all observations after storage which might be due 
to degradation of carotenoids by oxidation leading to declined in carotenoid content during storage. Kinetics of colour changes in dehydrated blanched and unblanched carrots during storage followed a first order kinetics for thermal degradation of $\beta$ carotene. Oxidation lead to the formation of highly reactive species, such as alkyl and peroxyl radicals, which could, in turn, increase the degradation of easily oxidizable compounds (Koca et al., 2007). Stability, however, was also affected by the presence of endogenous antioxidants, such as tocopherols and tocotrienols. Studies performed in non-polar environments showed that tocopherols could protect $\beta$-carotene from oxidative damage (Krinsky and Yeum, 2003). At lower temperature leutin degradation was slow in sunflower oil because of the higher content of tocopherols. Due to increased temperature, the protection offered by tocopherol might be overcome by formation of relative species caused by lipid peroxidation (Hojnik et al., 2008).

In conclusion, after a concise study it was observed that combination of packaging materials, seasons and varieties was found to be effective in extending the post harvest life of all varieties of marigold. The results confirmed that shelf life of loose marigold flowers stored during mid winter season (18$20^{\circ} \mathrm{C}$ and 60 to $75 \% \mathrm{RH}$ ) in plastic baskets could be extended for 12 days in Bidhan 3, with acceptable marketable quality. Our results have immense potentials to offer appropriate methods for storing flowers even for short term to provide solution during unforeseen circumstances. Even short term storage (for 10 - 12 days) can harvest the possibility to earn more and resist unwanted losses.

\section{Acknowledgement}

We thank Dr. R Sadhu Khan, P.I. AICRP on Floriculture, Bidhan Chandra Krishi
Vishvavidyalaya, Mohanpur, Nadia, West Bengal, India for all the support and corporation extended towards us during our study.

\section{References}

Ahmad, I., Asif, M., Amjad, A. and Ahmad, S. 2011. Fertilization enhances growth, yield, and xanthophyll contents of marigold. Turk J. Agric. For., 35: 641648.

Anon. 2007. Annual report. AICRP on Floriculture. Bidhan Chandra Krishi Viswavidyalaya Mohanpur, Nadia, West Bengal.

Bhattacharjee, S.K. 2001. Post harvest management of flowers. In: Hand book of Horticulture. (Ed.). Chadha, K.L. IARI, New Delhi, India, pp. 969-981.

Bose, T.K. and Raghava, S.P.S. 1975. Low temperature prolongs the storage life jasmine flower. Sci. Culture, 41: 115116.

Croots, G.D. 1973. Internal metabolic changes in cut flowers. Hort Sci., 8: 195-198.

Hemalatha, G., Jasmine, J.A. and Ponnuswamy, V. 2000. Prepackaging studies in KKH-1 brinjal. Indian $J$. Nutr. Dietet., 37: 365-369.

Hojnik, M., Skerget, M. and Knez, Z. 2008. Extraction of lutein from marigold flower petals-experimental kinetics and modeling. LWT Food Sci. Technol., 41: 2008-2016.

Koca, N., Burdurlu, H.S. and Karadeniz, F. 2007. Kinetics of colour changes in dehydrated carrots. J. Food Eng., 78(2): 449-455.

Krinsky, I.N. and Yeum, K.J. 2003. Carotenoid radical interactions. Biochem. Biophysic. Res. Comm., 305(3): 754-760.

Leopold, A. and Kriedemann, P. 1975. Plant growth and development, McGraw-Hill, USA. 
NHB. 2012. National horticulture Board. In: Indian Horticulture Database (Ed. Bijay Kumar). Ministry of Agriculture, Government of India 85, Institutional Area, Gurgaon India, pp- 296.

Nichols, R. 1966. Ethylene production during senescence of flowers. J. Horti. Sci., 41: $279-290$.

Nichols, R. 1975. Seneccence and sugar status of the cut flower, Acta Horti., 41: 21-29

Ranganna, S. 2000. Manual of analysis of fruits and vegetable products. Tata Mc. Graw Hill Publishing Company Ltd. West Patel Nagar, New Delhi-8.

Rogers, M.J. 1973. Photosynthesis and respiration of Arceuthobium tsugense. Portland State University, Portland.

Walters, W.E. 1966. Influence of post harvest handling techniques on vase life of gladiolus flowers, Proceedings of Florida Horti. Sci., 79: 456-459.

\section{How to cite this article:}

Preema Devi, M., Laishram Hemanta, Rocky Thokchom, S. Chakrabarty and Dhua, R.S. 2017. A Study on Different Packaging Materials Affect Shelf Life of Marigold at Different Harvesting Seasons under West Bengal Condition. Int.J.Curr.Microbiol.App.Sci. 6(8): 16651673. doi: https://doi.org/10.20546/ijcmas.2017.608.200 